\title{
Revisión conceptual de "calidad educativa" y sus procesos en las instituciones de educación superior de criminología
}

\section{Conceptual review of "educational quality" and its processes in criminology higher education institutions}

Wael Sarwat Hikal Carreón. ${ }^{1}$

\begin{abstract}
.
DOI: https://doi.org/10.33262/cienciadigital.v4i3.1312

This article is part of the doctoral research "Study of Relevance of the Training and Professional Exercise Projects of Criminologists" at the Autonomus University of New Lion (AUNL), funded by the National Council of Science and Technology (NCST), which studies the quality accreditation process in the centers of higher education of criminology and criminalistic in Mexico. The objective of the present is review the concepts of educational quality and discover their characteristics. The method used consists in the review of quality documents in education, on the other hand, institutions that are already accredited under the standards of the evaluating bodies, are reviewed. With the revision of these concepts, it is the proposal to implement standards in schools to join the trend of improvement in pedagogical processes, infrastructure, auditing, etc., for the continuous strengthening of education.
\end{abstract}

Keywords: Educational education; Educational efficiency; Educational input; Educational relevance.

\section{Resumen.}

El presente artículo es parte de la investigación doctoral "Estudio de Pertinencia de los Proyectos de Formación y Ejercicio Profesional de los Criminólogos" en la Universidad Autónoma de Nuevo León (UANL), financiada por el Consejo Nacional de Ciencia y Tecnología (CONACYT), que estudia el proceso de

${ }^{1}$ Universidad Autónoma de Nuevo León, Facultad de Filosofía y Letras, wael.hikalcrr@uanl.edu.mx 
acreditación de calidad en los centros de educación superior de criminología y criminalística en México. El objetivo del presente del es revisar los conceptos de calidad educativa y descubrir sus características. El método empleado consiste en la revisión de documentos sobre calidad en la educación, por otro lado, se revisan las instituciones que se encuentran ya acreditadas bajo los estándares de los organismos evaluadores. Con la revisión de estos conceptos, resulta la propuesta de implementar estándares en los centros escolares para unirse a la tendencia de mejora en los procesos pedagógicos, infraestructura, auditoría, etcétera, para el fortalecimiento continuo de la educación.

Palabras clave: Aporte educacional; Calidad de la educación; Eficiencia de la educación; Pertinencia de la educación.

\section{Introducción.}

Este trabajo presenta diversos conceptos de calidad educativa para relacionarlos para con los centros escolares que imparten criminología y criminalística a nivel superior, para promover la reflexión sobre sus características, objetivos y procesos de cambio que implican estudios de condición de las áreas de la escuela, con miras a identificar su nivel, ya sea para mantenerlo o trabajarlo para mejorarlo, con ello adquirir la etiqueta de "centro acreditado". Los métodos empleados son el de análisis de textos, mediante este se revisan diversos textos enfocados a la reflexión de la calidad desde el ámbito empresarial que luego se adaptó al nivel escolar, donde diversos parámetros de mejora en los procesos y producción, así como hacer bien el procedimiento, se integraron a los centros escolares para evaluar su rendimiento, desde enfoques de calidad de un modo incluyente, transformador, capacitador de personas para una ciudadanía colectiva, y en otros aspectos contemplando la creación de mejores productos (egresados) competentes, líderes, empresarios. También se revisan los órganos acreditadores en materia educativa en México, para mostrar qué centros escolares en la materia de la presente investigación, están acreditados. Del análisis de los conceptos y su desmenuzamiento, resulta la propuesta de vincular los procesos de calidad educativa en los centros escolares de criminología y criminalística, lo que mejoría su percepción a nivel social, en prestigio, presencia, estrategias pedagógicas, satisfacción entres los alumnos, padres de familia, programas educativos y currículos formativos, infraestructura, evaluación del aprendizaje, resultado social en impacto a nivel intervención para la atención a las necesidades, así como absorción laboral, etcétera.

\section{Método.}

El método utilizado es el análisis de texto (Sales Garrido, 2003), a través de la lectura comprensiva de documentos que hacen énfasis en la calidad educativa, para descomponerlos en sus partes, luego sintetizarlos y orientarlos a su aplicación para los campos de la criminología y criminalística. Se realiza la lectura y relectura de los textos con el fin de establecer los referentes clave que construyen la calidad educativa (Acuña Gamboa y Pons Bonals, 2016). El criterio de selección de lecturas, se realiza consultando los índices Scielo, Redalyc y Dialnet, tomando de referencia los resultados que arrojan, donde de cuatro referencias, tres coinciden en las primeras dos plataformas, mientras que en la última, fue el primer resultado arrojado (tabla 1). Además de buscar y localizar los órganos que en México, fungen como acreditadores de calidad a nivel educación superior 
(tabla 2). Dicha información figura en la institución nacional que da la rectoría de la educación en el país, que a su vez, es la que autoriza y vigila a los órganos acreditadores. Se plantea el problema de las definiciones de calidad y su entrelazar con las áreas mencionadas, se trata de explicar la situación planteada, se define el trabajo que se podrán realizar, en la articulación lógica se presentan las áreas a reforzar mediante la presentación de hipótesis que atribuyen a la calidad, mejoras en los sistemas educativos.

Tabla 1. Literatura revisada.

\begin{tabular}{llc}
\hline Autoría & Título & Año \\
\hline $\begin{array}{l}\text { Acuna Gamboa, L.A. } \\
\text { y Pons Bonals, L. }\end{array}$ & $\begin{array}{l}\text { Calidad educativa en México. De las } \\
\text { disposiciones internacionales a los remiendos del } \\
\text { proyecto nacional }\end{array}$ & 2016 \\
$\begin{array}{l}\text { Vásquez Tasayco, A. } \\
\text { Rodríguez Arocho, }\end{array}$ & $\begin{array}{l}\text { Calidad y calidad educativa } \\
\text { El concepto de calidad educativa: Una mirada } \\
\text { W. }\end{array}$ & 2013 \\
$\begin{array}{l}\text { Mítica desde el enfoque históricocultural } \\
\text { F.M. }\end{array}$ & $\begin{array}{l}\text { La calidad de la educación y las variables de } \\
\text { proceso y de producto }\end{array}$ & 1995 \\
\hline
\end{tabular}

Fuente: Elaboración propia.

Para el análisis de los textos seleccionados, se revisaron los conceptos que apuntan de diversos autores, así como de reflexión de los propios autores, paráfrasis y comentarios. La sistematización de estos, permitió identificar características de la calidad educativa, como infraestructura, equipamiento, docentes, formación, programas de estudio. De la lectura de los organismos acreditadores, permite ubicar cuáles son los autorizados por la Secretaría de Educación Pública (SEP), a nivel general, descartando organismos sobre conocimientos particulares.

\section{Discusión.}

La calidad es un término presente en diversos aspectos de la vida, se habla de calidad en los servicios de salud, en el estilo de vida, calidad en productos, servicios, etcétera, esta es un marco referencial para direccionar la producción y prestación de servicios, donde también, existen clasificaciones, donde se trata de diferentes tipos de calidad o niveles, siendo parámetros de medición y selección, ya sea por usuarios, consumidores, evaluadores, medidores de calidad, etcétera. Mario De Miguel (1995) menciona la necesidad de construir unos indicadores de calidad objetivables, precisos y seguros porque la evaluación interna es el único instrumento que permite detectar trayectorias erróneas y corregirlas en la dirección marcada" (p. 3.). También con "base en el diseño de perfiles, parámetros e indicadores" (Acuña Gamboa y Pons Bonals, 2016, p. 167). Esto es puesto que vivimos en un ambiente de la calidad o cultura de calidad.

En la calidad se establecen criterios de satisfacción y precios, si se compara un hotel con otro según su costo, se espera que tenga mejores cualidades que el otro de menor precio. Lo mismo en la ropa, la marca asignada a las prendas antepone que puede ser de calidad, de ahí también la presunción de estas, puesto que en general, aquello que se asume de calidad, genera estatus, prestigio, reconocimiento, las empresas invierten en su calidad, para garantizar sus ingresos, producción y aseguramiento de nivel (Vásquez Tasayco, 
2013). A nivel educacional, si se trata de centros escolares privados, como por ejemplo el Instituto Tecnológico de Estudios Superiores de Monterrey (ITESM) o la Universidad Iberoamericana (IBERO) u otra, se asume que cuenta con regímenes de calidad más altos, o en contraste, escuelas privadas poco conocidas, de las cuales se pone en duda su validez, o podría ser similar la percepción sobre una escuela pública, donde tal vez por pagar cuotas bajas o no pagar, da motivo a un vaivén de interpretaciones entre buenas y malas sobre su calidad. Por lo tanto, los conceptos de calidad, se construyen según los intereses de cada caso concreto.

El caso que ocupa la presente investigación aborda los conceptos de calidad dirigidos a las instituciones de educación superior, que según los intereses de los clientes (padres de familia o alumnos) es relativo, tal vez una persona esté enfocada en la calidad teniendo de criterio los intercambios académicos, o las bibliotecas digitales, incluso el contar con estacionamiento para alumnos, docentes, o canchas, o auditorios, la realización de eventos, su intervención social, el establecimiento de becas, el contar con cuartos de renta para los alumnos, cafetería, restaurante, librería, cajero automático, o el mismo banco al interior de la escuela, de tal modo, la calidad se va construyendo con base a ideales y comparaciones. Si estos factores contribuyen a una matrícula más grande, entonces se entra en una obsesión por ofrecer más, para ganar más. Pero la calidad, va más allá de ello, que pueden ser vistas como trampas de la calidad (Santos Guerra, 1999).

Señala Rodríguez Arocho que "la calidad educativa es entendida de forma multidimensional y contextual. Más allá de los índices de matrícula, retención y aprovechamiento académico" (2010, p. 18). Asimismo, apunta que no debe ser entendida en términos de inversión y beneficio, traducido en beneficio económico, donde se trabajan los deseos del alumno y se le convierte en clientela, donde se recorta el tiempo de formación, exigencia, se da una preparación técnica, poco reflexiva, dejando de lado la alusión a la historia, fundamentación, donde se crean operadores, y no constructores de la realidad, se enfoca a que tener educación es un paradigma de obtener un lujo. Dado que la educación al privatizarse, pasó a ser parte del mundo de los negocios.

En la reflexión del autor antes mencionado (Rodríguez Arocho, 2010, p. 12), parte de los procesos de la calidad, son: Inspeccionar, asegurar la calidad, mantener el nivel, con esto se tiene una "calidad total", el primero entendido como la evaluación de la producción o los procesos de producción, el segundo, como, una vez identificados, descritos y controlados los procesos, se puede trabajar en el perpetuar las actividades inherentes, finalmente, con el ciclo continuo, se tiende a mantener tal nivel. En calidad total, se asumiría como la satisfacción de deseos y expectativas de los clientes. Esto, si se aplica al centro escolar, procura atender a los intereses particulares de la población, puede que incluso la calidad sea vista desde un aspecto tan sencillo, pero difícil como el tener agua en la escuela. "Esta situación ha generado, recientemente, una incisiva crítica a las orientaciones y a las formas actuales de la evaluación en el campo educativo" (Rodríguez Arocho, 2010, p. 15).

La calidad puede ser vista también como un proceso de concientización y reflexión permanente, si bien es un modelo, estándar a alcanzar, este no se agota al lograrlo, sino que se tiene que mantener, puesto que la sociedad cambia, y las demandas también, la calidad educativa debe adaptarse a los nuevos requerimientos. Tal vez como no antes, actualmente existe una necesidad de resolver los problemas sociales de violencia, por lo que ha llevado a que los centros escolares volteen su atención a las materias criminales, como criminología, psicología criminal, ciencias forenses, entre otras. Así "en el proceso se crea a la convicción de que están haciendo lo correcto por promover el desarrollo y la educación” (Rodríguez Arocho, 2010, p. 17). 
La calidad también es vista "como lo propio de algo que lo hace diferente" (Vásquez Tasayco, 2013, p. 49), pone en relieve la funcionalidad en relación a los elementos que conducen a la eficacia en los sistemas, y poner atención en el producto, en el que se vierte el resultado de la funcionalidad y se mide con la satisfacción o el nivel de complacencia. Desmenuza que, en el proceso de calidad, se parten en partes los elementos que dan lugar a tal, y de lo formado por la relación de sus elementos de manera lógica, muestran lo diferente y específico de ese total, lo que también se entiende como el desarrollo de la construcción de una unidad, en este caso, calidad educativa.

Este autor (Vásquez Tasayco, 2013) hace referencia al concepto de calidad desde la Real Academia Española, que ofrece diversos conceptos: "Propiedad o conjunto de propiedades inherentes a algo, que permiten juzgar su valor"; por ejemplo, esta escuela es de buena calidad. También la define como "superioridad o excelencia", por ejemplo, cuando se hace referencia a los alumnos de excelencia, medido su aprovechamiento o resultados con base a calificaciones o actividades, otro concepto también apegado a los intereses de esta investigación es: "Adecuación de un producto o servicio a las características especificadas" (Real Academia Española, 2020).

Del modo anterior, a la hipótesis aquí trabajada, un centro escolar se mide como de calidad cuando ha cumplido con el conjunto de propiedades propiamente establecidas por los órganos encargados de la evaluación, que, mediante exámenes rigurosos, se diagnostican las condiciones, emiten recomendaciones y de aprobarse se da el juicio de acreditación, lo que le distingue de otras por su excelencia en los estándares, al haber adecuado sus procesos y productos a lo solicitado. Los elementos de la calidad desde un enfoque de sistema serían (Vásquez Tasayco, 2013, p. 53): Insumos (infraestructura, equipamiento, docentes, personal administrativo, libros, espacios adecuados, laboratorios, bibliotecas), procesos (auditorías), productos (programas educativos, alumnos), dinámica (relaciones que permiten adquirir compromisos, planeación docente), redes de calidad interna (quienes ayudan a lograr objetivos) y externa (organismos de normalización) (Acuña Gamboa y Pons Bonals, 2016, p. 171).

Por otra parte, Alonso (citado en Vásquez Tasayco, 2013, p. 59) menciona como factores de la calidad educativa: Objetivos y fines, pertinencia y relevancia, procesos de aprendizaje, docentes, insumos, sistemas óptimos, y medios de evaluación. La calidad educativa vendrá a ser el resultado de la funcionalidad de los factores conforme se integren. El efecto de esto, dimensiona a la educación de calidad con efectos en: Excelencia en el saber, perfeccionamiento, producto (egresados eficientes), satisfacción de los usuarios, y la capacidad para atender a las demandas sociales (currículo que recoja elementos culturales con ideología de reconstrucción social) (pp. 60 y 68). No precisamente que la cuantificación de estos elementos garantice la calidad, sino la profundidad en los cambios, vista esta como una necesidad de lograr metas.

La calidad también es vista con varias características, según Mario De Miguel (1995, pp. 34-36), la calidad como prestigio, entendida como la reputación que ostenta la institución que ha sido acreditada, diferenciada una de otra, por el juicio de expertos que la evaluaron. Calidad en función de recursos, en dos aspectos, visto como la que más ha invertido, obtiene efectos benéficos de acreditarse, programas educativos, profesores y alumnos de excelencia, lo que conlleva a que agentes externos canalicen su financiación a esta. Calidad como resultado, relacionado a lo anterior, los resultados de la puesta en función de los procesos de calidad, conduce a profesores, alumnos y programas educativos, mejores. Calidad como cambio, siendo este un valor agregado al tener más calidad que otros, logrando mejor incidencia en el ambiente social e interno escolar. "Las intuiciones de mayor calidad son aquellas que tienen más impacto sobre los conocimientos de los alumnos" (Astin citado por De Miguel, 1995, p. 35). 
Calidad como adecuación a propósitos, sus objetivos se adecuan al marco legal, a las necesidades y satisfacciones. Calidad como perfección, consiste en ese proceso de concientizar e internalizar los cambios, comprenderlos, ser consciente de sus beneficios e introducirlos en su cultura organizacional. De modo general, los aspectos de la calidad educativa están en: Formación docente, recursos educativos, investigación, orientación profesional, evaluación del sistema. Que resulta reflejado en el rendimiento del alumno, crecimiento en titulación del profesorado, medios de enseñanza, instalaciones y actividades, materias y fines, formación, vinculación del centro escolar con la comunidad (De Miguel, 1995, p. 36). La obtención de la acreditación, está en función de reproducir los requerimientos en base a resultados. Perspectivas más amplias incluyen evaluar el impacto social.

Según otro estudio realizado por Acuña Gamboa y Pons Bonals (2016, pp. 160 y 161), el Banco Interamericano de Desarrollo, la Organización para la Cooperación el Desarrollo Económico y la Organización de las Naciones Unidas para la Educación, la Ciencia y la Cultura, la calidad educativo se presenta a través de diversos atributos: Infraestructura y equipamiento escolar, evaluación del desempeño docente, y formación docente inicial y continua, los cuales se van tomando en adaptación a los diferentes niveles educativos y necesidades particulares. El estudio resalta que a México "se le considera como uno de los países en desarrollo que presenta los más bajos índices de aprovechamiento escolar en todos sus niveles educativos" (Acuña Gamboa y Pons Bonals, 2016, p. 162). Para concluir con los conceptos, a manera de unificar en un par de palabras el concepto y objetivo de la calidad, esta puede referirse a "hacer las cosas bien" Rodríguez Arocho (2010, p. 11), por otro lado: "calidad de la organización, calidad del curriculum y calidad de la enseñanza" (De Miguel, 1995, p. 42).

En el tema de los organismos normalizadores de la calidad, en México, la institución que vigila y controla el establecimiento de escuelas y programas educativos, es la SEP, la cual, a su vez, autoriza a organismos facultados y delegados para evaluar programas educativos y centros escolares, de manera general; es decir, en áreas de conocimiento por ciencias sociales, naturales, humanas, etcétera, así como sectorizadas por específicas, como medicina, derecho, psicología. Por parte de los posgrados, se ha facultado a un organismo descentralizado del gobierno federal para evaluar estos, con independencia de otras vinculaciones extranjeras que se puedan gestionar, el reconocimiento oficial, es el establecido por la SEP y las instituciones delegadas para tal efecto.

\section{Resultados.}

El análisis de los conceptos de calidad en la educación resulta una condición necesaria para promover la práctica reflexiva y continua, comprender de qué se trata a nivel diagnóstico de las condiciones que guarda el centro educativo, y las líneas de cambio a trabajar con base a las características, fines y objetivos de los estándares de calidad en la educación. No se especula sobre los procedimientos de mejora educativa con bases en la introducción de la calidad, sino que tiene una secuenciación lógica de actividades las cuales se ven establecidas por organismos evaluadores y acreditadores; es decir, agentes externos a la institución educativa, que, mediante parámetros específicos, auditan, sugieren y evalúan, en México, según el grado a evaluar, el programa educativo, entre otros, es la institución que le evaluara.

Se detecta un problema de orden ¿A qué campo del conocimiento corresponde la evaluación? La criminología y criminalística son en dos aspectos, conjuntos de significados, de primer orden, en ambos casos, "crimi" que se refiere a crimen y "logía" e "ística" que indica el estudio especializado hacía un crimen. Además de ello, ambas, en 
la práctica se componen de múltiples ciencias y campos que se ocupan de temas criminales; por ejemplo, la criminología se vale de la antropología, sociología, derecho, psicología, estadística, política, economía, etcétera. La criminalística, de la medicina, química, toxicología, balística, dactiloscopia, psiquiatría, computación, ingenierías, informática, entre otras.

Derivado de lo anterior ¿A qué ente corresponde su evaluación para acreditar la calidad? ¿A organismos de medicina? ¿A entidades de acreditación en sociología o derecho? Para evitar la polémica, aunque no debería ser punto de huida, puede apegarse a evaluadoras generales que revisan procesos que se basan en términos cotidianos para la educación; por ejemplo, infraestructura, planta docente, recursos didácticos, procesos, productos, pedagogía, etcétera. Imaginar cuan complejo es evaluar a los centros escolares de criminología y criminalística por la diversidad de materias que la construyen, da lugar a la ambigüedad. Rodríguez Arocho (2010) cuestiona: “¿Quién o quiénes crean los criterios mediante los cuáles luego se juzgará su valor? ¿Qué mecanismos y procesos se utilizarán para determinar la superioridad y la excelencia y con qué consecuencias?” (p. 9).

La respuesta puede encontrase en lo siguiente, la Secretaría de Educación Pública (2018) establece los organismos autorizados para evaluar programas educativos y centros escolares, para evaluación de posgrados, es el Consejo Nacional de Ciencia y Tecnología (CONACYT), estos dos, son entes públicos federales. Para programas educativos de licenciatura y otras características, están facultados por la SEP, el Consejo para la Acreditación de la Educación Superior (2020), Comités Interinstitucionales para la Evaluación de la Educación Superior (2019), y para la evaluación de conocimientos y competencias, es el Centro Nacional de Evaluación para la Educación Superior (2020), además de las particulares en cada campo del conocimiento, pero estos no se abordan aquí.

Tabla 2. Organismos acreditadores.

\begin{tabular}{|l|}
\hline \multicolumn{1}{|c|}{ Organismo } \\
\hline Centro Nacional de Evaluación para la Educación Superior \\
\hline Consejo Nacional de Ciencia y Tecnología \\
\hline Consejo para la Acreditación de la Educación Superior \\
\hline Comités Interinstitucionales para la Evaluación de la Educación Superior \\
\hline
\end{tabular}

Fuente: Elaboración propia.

La SEP cuenta con un padrón de programas educativos de licenciatura acreditados por los órganos antes señalados, de la búsqueda en el Programa Nacional de Programas Educativos de Calidad, indica que 7 están acreditados (tabla 3), mientras que programas educativos de posgrados avalados por CONACYT, son también 7 (tabla 4). En contraste a estas cifras, existen 527 programas educativos de grado técnico, licenciatura, en línea, especialidad, maestría y doctorado en materia criminal (Hikal Carreón, 2020).

Tabla 3. Programas educativos de licenciatura acreditados.

\begin{tabular}{|l|l|l|}
\hline \multicolumn{1}{|c|}{ Programa } & \multicolumn{1}{|c|}{ Institución educativa } & Certificación \\
\hline $\begin{array}{l}\text { Licenciatura en } \\
\text { Criminalística }\end{array}$ & $\begin{array}{l}\text { Centro de Estudios Universitarios Xochimilco } \\
\text { (plantel Rampa Yujimalinda) }\end{array}$ & COPAES \\
\hline $\begin{array}{l}\text { Licenciatura en } \\
\text { Criminalística }\end{array}$ & $\begin{array}{l}\text { Centro de Estudios Universitarios Xochimilco } \\
\text { (plantel calle Novena) }\end{array}$ & COPAES \\
\hline
\end{tabular}




\begin{tabular}{|c|c|c|}
\hline $\begin{array}{l}\text { Licenciatura en } \\
\text { Criminología }\end{array}$ & $\begin{array}{l}\text { Universidad Autónoma de Nuevo León, } \\
\text { Facultad de Derecho y Criminología }\end{array}$ & $\begin{array}{l}\text { COPAES } \\
\text { CIEES } \\
\text { CENEVAL }\end{array}$ \\
\hline $\begin{array}{ll}\text { Licenciatura en } \\
\text { Criminología }\end{array}$ & $\begin{array}{l}\text { Universidad Autónoma de Querétaro, Facultad } \\
\text { de Derecho }\end{array}$ & $\begin{array}{l}\text { CIEES } \\
\text { CENEVAL }\end{array}$ \\
\hline $\begin{array}{l}\text { Licenciatura en } \\
\text { Criminología }\end{array}$ & $\begin{array}{l}\text { Universidad Autónoma de Tamaulipas, Unidad } \\
\text { Académica Multidisciplinaria Reynosa-Aztlán }\end{array}$ & $\begin{array}{l}\text { CIEES } \\
\text { CENEVAL }\end{array}$ \\
\hline $\begin{array}{ll}\text { Licenciatura en } \\
\text { Criminología }\end{array}$ & Universidad Ixtlahuaca & CIEES \\
\hline $\begin{array}{ll}\text { Licenciatura } & \text { en } \\
\text { Criminología, } & \\
\text { Criminalística } & \text { y } \\
\text { Técnicas } & \\
\text { Periciales } & \\
\end{array}$ & $\begin{array}{l}\text { Colegio Libre de Estudios } \\
\text { (plantel Puebla) }\end{array}$ & CIEES \\
\hline
\end{tabular}

Fuente: Elaboración propia.

Tabla 4. Programas educativos de posgrado acreditados.

\begin{tabular}{|l|l|l|}
\hline \multicolumn{1}{|c|}{ Programa } & \multicolumn{1}{|c|}{ Institución educativa } & Nivel \\
\hline $\begin{array}{l}\text { Doctorado en } \\
\text { Criminología }\end{array}$ & $\begin{array}{l}\text { Universidad Autónoma de Nuevo } \\
\text { León, Facultad de Derecho y } \\
\text { Criminología }\end{array}$ & En desarrollo \\
\hline $\begin{array}{l}\text { Doctorado en Psicología } \\
\text { con Énfasis en Salud y } \\
\text { Violencia }\end{array}$ & $\begin{array}{l}\text { Universidad Autónoma de Ciudad } \\
\text { Juárez, Instituto de Ciencias Sociales y } \\
\text { Administración }\end{array}$ & En desarrollo \\
\hline $\begin{array}{l}\text { Maestría en Criminología } \\
\text { y Ciencias Forenses }\end{array}$ & $\begin{array}{l}\text { Universidad Autónoma de } \\
\text { Tamaulipas, Unidad Académica } \\
\text { Multidisciplinaria Reynosa-Aztlán }\end{array}$ & Consolidada \\
\hline $\begin{array}{l}\text { Maestría en Medicina } \\
\text { Forense }\end{array}$ & $\begin{array}{l}\text { Universidad Veracruzana, Instituto de } \\
\text { Medicina Forense }\end{array}$ & Consolidada \\
\hline Maestría en Valuación & $\begin{array}{l}\text { Universidad Autónoma de Nuevo } \\
\text { León, Facultad de Arquitectura }\end{array}$ & En desarrollo \\
\hline $\begin{array}{l}\text { Especialidad en Familias y } \\
\text { Prevención de la Violencia }\end{array}$ & $\begin{array}{l}\text { Universidad Autónoma de Querétaro, } \\
\text { Facultad de Ciencias Políticas y } \\
\text { Sociales }\end{array}$ & En desarrollo \\
\hline $\begin{array}{l}\text { Especialidad en Género, } \\
\text { Violencia y Políticas } \\
\text { Públicas }\end{array}$ & $\begin{array}{l}\text { Universidad Autónoma del Estado de } \\
\text { México, Facultad de Ciencias Políticas } \\
\text { y Sociales }\end{array}$ & En desarrollo \\
\hline
\end{tabular}

Fuente: Elaboración propia.

Una trampa en la interpretación de los conceptos y procesos de calidad educativa viene asimilada a la cantidad de ingreso de alumnos; es decir, no porque se tenga calidad, implicará mayor cuerpo estudiantil, tampoco lo contrario, en una escuela donde hay muchos alumnos, no indica que sea la mejor, lo "mejor" y la "calidad", el establecimiento de estas profesiones debe atender a lo que la sociedad violentada necesita, no a una moda comercial de "lo que los jóvenes quieren estudiar". Se mide por los criterios que establecen las acreditadoras, teniendo estas la facultad de evaluar, sobre una previa 
autorización gubernamental, peligraría tal vez, el día que se suelte al sector privado el autorregularse para evaluar y acreditar, puesto que no habría control estricto de los parámetros, la credibilidad aumenta cuando la evaluación se realiza por ajenos, por gente no familiarizada con los dueños de las escuelas, ni con el personal, ni por amistad o simpatía.

\section{Propuestas.}

Se postula que con un modelo pedagógico y organizacional, metodológico, sugerido para los centros de enseñanza de la criminología, criminalística, entre otras, permitirá mejorar los procesos educativos con miras a un mejor impacto social, profesional, en general, de resultados (Oficina Regional de Educación de la UNESCO para América Latina y el Caribe, 2008, p. 64). Este proceso llevas inherente varios apartados:

- Mejorar la calidad de enseñanza docente, pues como indican Chávez González y Benavides Martínez: "En la educación superior, orientada a la formación de profesionales, al igual que ocurre en otros niveles educativos, la participación de los profesores es fundamental para el logro de los objetivos, debido a su papel central como representantes del orden institucional; (...)" (2011, p. 2).

- Legitimar la profesión: A través del dominio de las competencias, habilidades, técnicas, saberes, investigación, desarrollo del conocimiento, útil para la sociedad, resolver sus problemas, así como útil para el profesional, capaz de emplearse, auto emplearse, argumentar, investigar (Oficina Regional de Educación de la UNESCO para América Latina y el Caribe, 2008, p. 43).

- Aproximar a la escuela con las empresas e instituciones públicas: Establecer una red que conecte a los centros de aprendizaje con los sectores públicos y privados para la facilitación del empleo y prácticas de los estudiantes y egresados (Comisión Europea, 1996, p. 10; Oficina Regional de Educación de la UNESCO para América Latina y el Caribe, 2008, pp. 92).

- Fomentar la investigación científica para lograr respuestas a las incógnitas de los problemas de la violencia "es también un saber disponible, una actitud de los sujetos ante la realidad-, y supone que el alumnado tiene preguntas, tiene capacidad de buscar respuestas, de buscar soluciones a problemas de diversa naturaleza que su despertar a la vida y a la experiencia comunitaria les plantean" (Bonifacio Barba, 2018, p. 969).

\section{Conclusiones:}

Del análisis de los conceptos de calidad en la educación y su relación propositiva para con las escuelas que impartan criminología y criminalística, no debe considerarse que el introducirse a la cultura de calidad educativa sea vista como un problema, sino como toma de conciencia de autodiagnóstico para conocer qué se tiene y a qué se puede aspirar para ser mejor, sin llegar a un punto de confort, sino de procesamiento permanente, que permitirá destacar las cualidades obtenidas y trabajadas desde la óptica de ser mejor en comparación con otros centros escolares en la materia, no para tomar una postura clasista o discriminadora, la esencia en la mejora de los procesos es capacitar mejor a los futuros profesionales con base a las necesidades sociales, para que sean agentes transformadores de mejoras de las condiciones de una comunidad, así la violencia, los grupos vulnerables, los sistemas de justicia, de investigación pericial, de prevención del delito, requieren 
criminólogos y criminalistas comprometidos con las problemáticas que habrán de estudiar para planificar intervenciones. También, la calidad educativa, se ve como secuencialmente a la inclusión y satisfacción laboral de los egresados de las escuelas.

\section{Referencias bibliográficas:}

Acuña Gamboa, L.A. y Pons Bonals, L. (2016). Calidad educativa en México. De las disposiciones internacionales a los remiendos del proyecto nacional. Revista Internacional de Investigación en Ciencias Sociales, 12(2), 155-174. Recuperado de http://scielo.iics.una.py/pdf/riics/v12n2/2226-4000-riics-12-0200155.pdf

Bonifacio Barba, J. (2018). La calidad de la educación. Los términos de su ecuación. Revista Mexicana de Investigación Educativa. 23(78), 963-979. Recuperado de https://www.comie.org.mx/revista/v2018/rmie/index.php/nrmie/article/view/118 $4 / 1167$

Centro Nacional de Evaluación para la Educación Superior (2020). Perfil institucional. Recuperado de https://www.ceneval.edu.mx/perfil-institucional

Chávez González, G. y Benavides Martínez, B. (2011). Los profesores universitarios: entre ala exigencia profesional y el compromiso ético-social.37. Sinéctica. Revista Electrónica de Educación. Recuperado de http://www.scielo.org.mx/pdf/sine/n37/n37a3.pdf

Consejo Nacional de Ciencia y Tecnología (2019). Programa Nacional de Posgrados de Calidad. Recuperado de https://www.conacyt.gob.mx/index.php/becas-yposgrados/programa-nacional-de-posgrados-de-calidad

Consejo Nacional de Ciencia y Tecnología (2018). Programa Nacional de Posgrados de Calidad. Recuperado de https://www.conacyt.gob.mx/index.php/becas-yposgrados/programa-nacional-de-posgrados-de-calidad

Consejo para la Acreditación de la Educación Superior (2020). Proceso de acreditación. Recuperado de https://www.copaes.org/procesoacreditacion.html

Comités Interinstitucionales para la Evaluación de la Educación Superior (2019). Acreditación institucional. Recuperado de https://www.ciees.edu.mx/acreditacion/

Hikal Carreón, W.S. (2020). Censo de centros escolares y programas educativos en criminología, criminalística, victimología y carreras afines en México. Archivos de Criminología, Seguridad Privada y Criminalística, 8(15). Recuperado de https://drive.google.com/file/d/13cs7uy8-Okezddai4Bd94IH1ENISPtmO/view

Miguel Díaz, De, F.M. (1995). La calidad de la educación y las variables de proceso y de producto. Ikastaria: Cuadernos de Educación, 8, 29-52. Recuperado de http://www.euskomedia.org/PDFAnlt/ikas/08/08029051.pdf 
Oficina Regional de Educación de la UNESCO para América Latina y el Caribe (2008). Educación y Diversidad Cultural: Lecciones desde la Práctica Innovadora en América Latina. Chile: Organización de las Naciones Unidas para la Educación, la Ciencia y la Cultura Recuperado de https://unesdoc.unesco.org/ark:/48223/pf0000162699

Sales Garrido, L.M. (2003). La comprensión, el análisis y la construcción de textos según el enfoque comunicativo. Universidades, 25, 13-33. Recuperado de https://www.redalyc.org/pdf/373/37302503.pdf

Santos Guerra, M.Á. (1999). Las trampas de la calidad. Acción Pedagógica, 8(2), 78-81. Recuperado de https://dialnet.unirioja.es/descarga/articulo/2973330.pdf

Secretaría de Educación Pública (2018). Padrón Nacional de Programas Educativos de Calidad. Recuperado de https://www.pnpec.sep.gob.mx/

Secretaría de Educación Pública (2018). Comunicado 65.- Acuerdan SEP y organismos acreditadores trabajar para configurar un nuevo paradigma de evaluación y la acreditación. Recuperado de https://www.gob.mx/sep/es/prensa/comunicado65-acuerdan-sep-y-organismos-acreditadores-trabajar-para-configurar-unnuevo-paradigma-de-evaluacion-y-la-acreditacion-de-programas-de-nivelsuperior?idiom $=\mathrm{es}$

Real Academia Española (2020). Diccionario de la Lengua Española. Madrid. Recuperado de https://dle.rae.es/calidad?m=form

Rodríguez Arocho, W. (2010). El concepto de calidad educativa: Una mirada crítica desde el enfoque históricocultural. Revista Electrónica "Actualidades Investigativas en Educación”, 10(1), 1-28. Recuperado de https://www.redalyc.org/articulo.oa?id=44713068015

Vásquez Tasayco, A. (2013). Calidad y calidad educativa. Investigación Educativa. 17(2), 49-71. Recuperado de https://revistasinvestigacion.unmsm.edu.pe/index.php/educa/article/view/8206/ 7157

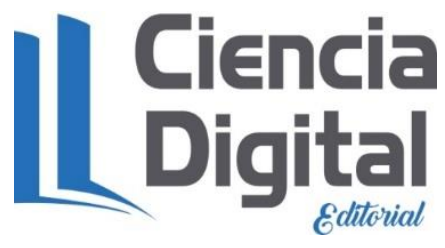




\section{PARA CITAR EL ARTÍCULO INDEXADO.}

Hikal Carreón, W. S. (2020). Revisión conceptual de "calidad educativa" y sus procesos en las instituciones de educación superior de criminología. Ciencia Digital, 4(3), 152-163. https://doi.org/10.33262/cienciadigital.v4i3.1312

\section{¿Ciencia}

El artículo que se publica es de exclusiva responsabilidad de los autores y no necesariamente reflejan el pensamiento de la Revista Ciencia Digital.

El artículo queda en propiedad de la revista y, por tanto, su publicación parcial y/o total en otro medio tiene que ser autorizado por el director de la Revista Ciencia Digital.
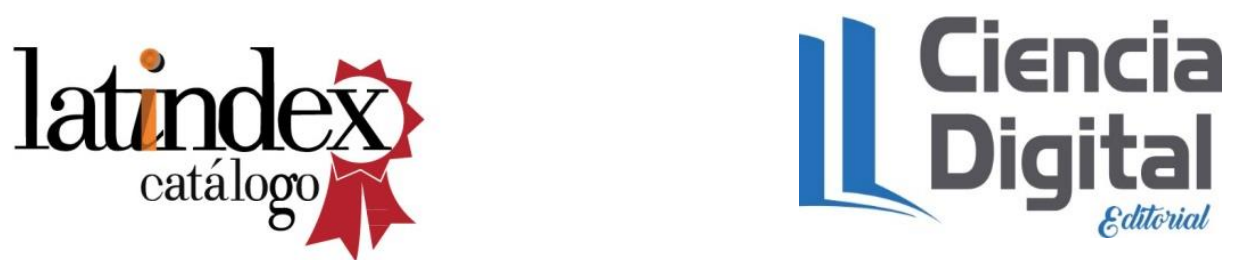